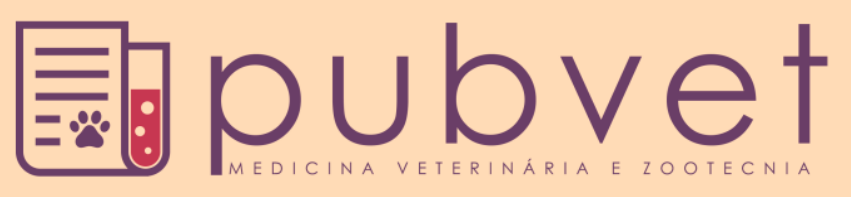

HTTP://DX.DOI.ORG/10.22256/PUBVET.V11N11.1163-1168

\title{
Acúmulo de forragem e morfogênese de Megathyrsus maximus cv. Mombaça sob níveis de fósforo
}

\author{
Newton de Lucena Costa ${ }^{1 *}$, Liana Jank², João Avelar Magalhães ${ }^{3}$, Fabíola Helena dos \\ Santos Fogaça ${ }^{4}$, Antônio Neri Azevedo Rodrigues ${ }^{5}$, Francisco José de Seixas Santos ${ }^{6}$

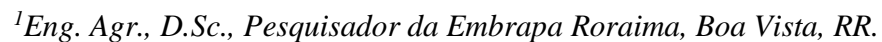 \\ ${ }^{2}$ Eng. Agr., Ph.D., Pesquisadora da Embrapa Gado de Corte, Campo Grande, MS. \\ ${ }^{3}$ Méd. Vet., Pesquisador da Embrapa Meio-Norte, Parnaíba, PI. \\ ${ }^{4}$ Zootecnista, D.Sc., Pesquisadora da Embrapa Meio-Norte, Parnaíba, PI. \\ ${ }^{5}$ Eng. Agr., D.Sc., Professor do Instituto Federal de Rondônia, Colorado do Oeste, RO. \\ ${ }^{6}$ Eng. Agr., D.Sc., Pesquisador da Embrapa Meio-Norte, Parnaíba, PI. \\ *Autor para correspondência, E-mail: newton.lucena-costa@embrapa.br
}

\begin{abstract}
RESUMO. O efeito de níveis de fósforo $\left(0,30,60,90\right.$ e $\left.120 \mathrm{~kg}^{\mathrm{de}} \mathrm{P}_{2} \mathrm{O}_{5} \mathrm{ha}^{-1}\right)$ sobre a produção de forragem e características morfogênicas e estruturais de Megathyrsus maximus foi avaliado em condições de campo. A adubação fosfatada afetou positiva e significativamente a produção de matéria seca verde (MSV), teores de $\mathrm{P}$, densidade populacional de perfilhos (DPP), número de folhas perfilho ${ }^{-1}$ (NFP), tamanho médio de folhas (TMF), índice de área foliar (IAF) e taxas de aparecimento (TAF), expansão (TEF) e senescência das folhas (TSF). Os máximos rendimentos de MSV, TAF, TEF, DPP, NFP, IAF e TMF foram obtidos com a aplicação de 96,1; 58,6; 69,8; 97,5; 64,4; 75,6 e 79,2 kg de $\mathrm{P}_{2} \mathrm{O}_{5} \mathrm{ha}^{-1}$, respectivamente. O nível crítico interno de $\mathrm{P}$, relacionado com $80 \%$ da produção máxima de MSV, foi estimado em $1,692 \mathrm{~g} \mathrm{~kg}^{-1}$. A eficiência de utilização de $P$ foi inversamente proporcional às doses de $\mathrm{P}$ aplicadas.
\end{abstract}

Palavras chave: Folhas, matéria seca, perfilhamento, senescência

\section{Forage accumulation and morphogenesis of Megathyrsus maximus cv. Mombaça under phosphorus levels}

\begin{abstract}
The effect of phosphorus levels $\left(0,30,60,90\right.$ and $120 \mathrm{~kg}$ of $\left.\mathrm{P}_{2} \mathrm{O}_{5} \mathrm{ha}^{-1}\right)$ on green dry matter (GDM) yield and morphogenetic and structural characteristics of Megathyrsus maximus cv. Mombaça was evaluated under field conditions. Phosphorus fertilization increased significantly GDM yields, $\mathrm{P}$ concentrations, density population of tillers (DPT), number of leaves plant ${ }^{-1}$ (NLP), medium blade length (MBL), leaf area index (LAI), leaf senescence rate (LSR), leaf appearance (LAR) and elongation rates (LER). Maximum GDM yields, LAR, LER, NT, NLT, LAI and MBL were obtained with the application of $96.1 ; 58.6 ; 69.8 ; 97.5 ; 64.4 ; 75.6$ and $79.2 \mathrm{~kg}$ of $\mathrm{P}_{2} \mathrm{O}_{5}$ ha $^{-1}$, respectively. The $\mathrm{P}$ critic level, related to $80 \%$ of GDM maximum production, was estimated at $1.692 \mathrm{~g} \mathrm{~kg}$ ${ }^{1}$. The $\mathrm{P}$ efficiency utilization was inversely proportional to the $\mathrm{P}$ rates applied.
\end{abstract}

Keywords: dry matter, leaves, tillering, senescence

\section{Acumulación de forraje y morfogénesis de Megathyrsus maximus cv. Mombasa bajo niveles de fósforo}

RESUMEN. El efecto de los niveles de fósforo (0, 30, 60, 90 y $120 \mathrm{~kg}$ de P2O5 ha-1) sobre la producción de forraje y características morfogenéticas y estructurales de Megathyrsus 
maximus cv. Mombaça se evaluó en condiciones de campo. La fertilización fosfatada afectó positivamente y significativamente la producción de materia seca verde (MSV), las concentraciones de $\mathrm{P}$ en el tejido, la densidad poblacional de las macollas (DPM), el número de hojas macolla ${ }^{-1}$ (NFM), el tamaño medio de las hojas (TMH), el índice de área foliar (IAF) y tasas de aparición (TAF), expansión (TEF) y senescencia de las hojas (TSF). Los máximos rendimientos de MSV, TAF, TEF, DPM, NFM, IAF y TMH se obtuvieron con la aplicación de 96,1; 58,6; 69,8; 97,5; 64,4; 75,6 y 79,2 kg de $\mathrm{P}_{2} \mathrm{O}_{5} \mathrm{ha}^{-1}$, respectivamente. El nivel crítico interno de $\mathrm{P}$, relacionado con el $80 \%$ de la producción máxima de MSV, fue estimado en 1,692 $\mathrm{g} \mathrm{kg}^{-1}$. La eficiencia de uso de P fue inversamente proporcional a las dosis de $\mathrm{P}$ aplicadas.

Palabras clave: composición química, hojas, macollaje, materia seca, senescencia

\section{Introdução}

Em Roraima, os solos sob vegetação de cerrados são caracterizados por baixa fertilidade natural, caracterizados por elevada acidez, baixa capacidade de troca catiônica e altos teores de alumínio trocável, limitando a produtividade e persistência das pastagens cultivadas, refletindo negativamente no desempenho zootécnico dos rebanhos.

Na formação e manejo de pastagens cultivadas, o conhecimento dos fatores nutricionais limitantes ao crescimento de gramíneas forrageiras torna-se imprescindível para a formulação de práticas adequadas de adubação (Costa, 2004). Em ensaios exploratórios de fertilidade do solo realizados em Roraima foi constatado ser o fósforo (P) o nutriente mais limitante ao crescimento de diversas gramíneas forrageiras, notadamente Megathyrsus maximus cvs. Centenário, Massai, Tanzânia-1 e Vencedor, cuja deficiência implica em reduções significativas nos rendimentos e na qualidade de sua forragem (Barger et al., 2002, Costa et al., 2009). O P desempenha importante papel no desenvolvimento do sistema radicular e no perfilhamento das gramíneas, sendo indispensável à fotossíntese, síntese e degradação dos carboidratos, além de participar ativamente da respiração celular, influenciando o armazenamento, transporte e utilização da energia produzida no processo fotossintético, além de favorecer o crescimento do sistema radicular, o que contribui para maior absorção de água e nutrientes (Lemaire and Chapman, 1996). Considerando-se o elevado investimento na aquisição de fertilizantes fosfatados e sua importância relativa na composição dos custos de produção dos sistemas pecuários, torna-se necessário assegurar sua máxima eficiência, através da determinação das doses mais adequadas para o estabelecimento e manutenção das pastagens.
A morfogênese de gramíneas forrageiras tropicais durante seu crescimento vegetativo pode ser descrita por três variáveis: a taxa de aparecimento, a taxa de alongamento e a duração de vida das folhas, as quais, apesar de sua natureza genética, são fortemente influenciadas pelas condições ambientais (temperatura, luz, água e fertilidade do solo) e práticas de manejo. As interações destas variáveis determinam as características estruturais: número de folhas vivas perfilho $^{-1}$ (NFV), tamanho médio de folhas (TMF) e densidade de perfilhos, as quais irão determinar o índice de área foliar (IAF) que representa o aparato utilizado para a interceptação da radiação pelo dossel da pastagem (Lemaire and Agnusdei, 2000, Patês et al., 2007). O NFV é relativamente constante para cada espécie e constitui critério objetivo na definição dos sistemas de pastejo a serem impostos no manejo das forrageiras, sendo decorrente da taxa de aparecimento e a duração de vida das folhas, enquanto que a taxa de alongamento foliar condiciona o TMF (Nabinger and Carvalho, 2009). Desta forma, estudos de dinâmica do crescimento de folhas e perfilhos de gramíneas forrageiras perenes são importantes para a definição de estratégias de manejo específicas para cada gramínea forrageira ( et al., 2009).

Neste trabalho foram avaliados os efeitos da adubação fosfatada sobre a produção de forragem e morfogênese de Megathyrsus maximus cv. Mombaça, nos cerrados de Roraima.

\section{Material e Métodos}

O ensaio foi conduzido no Campo Experimental da Embrapa Roraima, localizado em Boa Vista, durante o período de maio a setembro de 2015, o qual correspondeu a uma precipitação acumulada de $1.203,8 \mathrm{~mm}$ e temperatura média mensal de $24,71^{\circ} \mathrm{C}$. O solo da área experimental é Latossolo Amarelo, textura média, com as 
seguintes características químicas, na profundidade de $0-20 \mathrm{~cm}: \mathrm{pH}_{\mathrm{H} 2 \mathrm{O}}=4,7 ; \mathrm{P}=1,8$ $\mathrm{mg} / \mathrm{kg} ; \mathrm{Ca}+\mathrm{Mg}=0,98 \mathrm{cmol}_{\mathrm{c}} \cdot \mathrm{dm}^{-3} ; \mathrm{K}=0,03$ $\mathrm{cmol}_{\mathrm{c}} \cdot \mathrm{dm}^{-3} ; \mathrm{Al}=0,58 \mathrm{cmol}_{\mathrm{c}} \mathrm{dm}^{-3} ; \mathrm{H}+\mathrm{Al}=2,64$ $\mathrm{cmol}_{\mathrm{c}} \cdot \mathrm{dm}^{-3}$. O delineamento experimental foi em blocos ao acaso com três repetições. Os tratamentos consistiram de cinco níveis de fósforo $\left(0,30,60,90\right.$ e $120 \mathrm{~kg}$ de $\left.\mathrm{P}_{2} \mathrm{O}_{5} \mathrm{ha}^{-1}\right)$, aplicados a lanço após a uniformização da pastagem e sob a forma de superfosfato triplo. O tamanho das parcelas foi de 2,0 x 2,0 m, sendo a área útil de 1,0 $\mathrm{m}^{2}$. A adubação de estabelecimento constou da aplicação de $90 \mathrm{~kg}$ de $\mathrm{N} \mathrm{ha}^{-1}$ e $60 \mathrm{~kg}$ de $\mathrm{K}_{2} \mathrm{O} \mathrm{ha}^{-1}$, sob a forma de ureia e cloreto de potássio, respectivamente. A aplicação do nitrogênio foi parcelada em duas vezes, sendo metade quando da roçada da pastagem, ao início do experimento, e metade decorridos 35 dias. Durante o período experimental foram realizados quatro cortes a intervalos de 35 dias.

Os parâmetros avaliados foram rendimento de matéria seca verde (MSV), eficiência de utilização de fósforo, densidade populacional de perfilhos $\mathrm{m}^{-}$ 2 (DPP), número de folhas perfilho ${ }^{-1}$ (NFP), taxa de aparecimento de folhas (TAF), taxa de expansão foliar (TEF), taxa de senescência foliar (TSF), tamanho médio de folhas (TMF) e índice de área foliar (IAF). A TAC, a TEF e a TAF foram calculadas dividindo-se o rendimento e MS, o comprimento acumulado de folhas e o número total de folhas no perfilho, respectivamente, pelo período de rebrota. O TMF foi determinado pela divisão do alongamento foliar total do perfilho pelo número de folhas. Para o cálculo da área foliar foram coletadas amostras de folhas verdes completamente expandidas, procurando-se obter uma área entre 200 e $300 \mathrm{~cm}^{2}$. As amostras foram digitalizadas e a área foliar estimada com o auxílio de planímetro ótico eletrônico (Li-Cor 3100C). Posteriormente, as amostras foram levadas à estufa com ar forçado a $65^{\circ} \mathrm{C}$ até atingirem peso constante, obtendo-se a MS foliar. A área foliar específica (AFE) foi determinada através da relação entre a área de folhas verdes e a sua MS $\left(\mathrm{m}^{2} / \mathrm{g}\right.$ MS foliar). $\mathrm{O}$ índice de área foliar (IAF) foi determinado a partir do produto entre a MS total das folhas verdes $\left(\mathrm{g}\right.$ de $\left.\mathrm{MS} / \mathrm{m}^{2}\right)$ pela $\mathrm{AFE}\left(\mathrm{m}^{2} / \mathrm{g}\right.$ de MS foliar). A TSF foi obtida dividindo-se o comprimento da folha que se apresentava de coloração amarelada ou necrosada pela idade de rebrota.

$\mathrm{O}$ nível crítico interno (NCI) de $\mathrm{P}$ foi determinado ajustando-se a equação de regressão para rendimento de MSV (variável dependente) e níveis de $\mathrm{P}$ (variável independente) (equação 1) e para teores de $\mathrm{P}$ como variável dependente dos níveis de $\mathrm{P}$ aplicados (equação 2). Através da equação 1 calculou-se a dose de $\mathrm{P}$ aplicada relativa a $80 \%$ do rendimento máximo de MSV, sendo este valor substituído na equação 2 .

Os dados foram submetidos à análise de variância e de regressão considerando o nível de significância de $5 \%$ de probabilidade. Para se estimar a resposta dos parâmetros avaliados aos níveis de fósforo, a escolha dos modelos de regressão baseou-se na significância dos coeficientes linear e quadrático, por meio do teste " $t$ ", de Student, ao nível de 5\% de probabilidade.

\section{Resultados e Discussão}

A adubação fosfatada afetou os rendimentos de MSV $(\mathrm{P}<0,05)$, sendo a relação quadrática e o máximo valor estimado com a aplicação de 96,1 $\mathrm{kg}$ de $\mathrm{P}_{2} \mathrm{O}_{5}$ ha $^{-1}(3.640 \mathrm{~kg}$ de MSV ha-1), o qual foi inferior aos relatados por Costa et al. (2007) para M. maximus cv. Centenário $\left(138,9 \mathrm{~kg}\right.$ de $\mathrm{P}_{2} \mathrm{O}_{5}$ ha $^{-}$ $\left.{ }^{1}\right)$ e Pennisetum purpureum cv. Cameroon $(157,1$ $\mathrm{kg}$ de $\mathrm{P}_{2} \mathrm{O}_{5}$ ha $\left.^{-1}\right)$. A eficiência de utilização do fósforo foi inversamente proporcional às doses utilizadas (Tabela 1), contudo a gramínea apresentou maior responsividade que a reportada por Costa (2004) para pastagens de $M$. maximus cv. Tobiatã $(51,5 ; 41,3$ e $33,7 \mathrm{~kg}$ de $\mathrm{MSV} / \mathrm{kg}$ de $\mathrm{P}_{2} \mathrm{O}_{5} \mathrm{ha}^{-1}$, respectivamente para doses de 40,80 e $120 \mathrm{~kg}$ de $\mathrm{P}_{2} \mathrm{O}_{5} \mathrm{ha}^{-1}$ ). Os rendimentos de MSV registrados foram superiores aos relatados por Costa (2004) para pastagens de M. maximus cv. Tanzânia, fertilizadas com $80 \mathrm{~kg}$ de $\mathrm{P}_{2} \mathrm{O}_{5} \mathrm{ha}^{-1} \mathrm{e}$ submetidas a diferentes frequências de corte (1.856; 2.587 e $3.009 \mathrm{~kg}$ de MSV ha ${ }^{-1}$, respectivamente para cortes a cada 21, 35 e 42 dias). Costa et al. (2015) avaliando os efeitos da adubação fosfatada $\left(0,50,100\right.$ e $200 \mathrm{~kg}$ de $\mathrm{P}_{2} \mathrm{O}_{5}$ $\mathrm{ha}^{-1}$ ), em $M$. maximus cv. Massai constataram máxima produção de forragem com a aplicação de $164,7 \mathrm{~kg}$ de $\mathrm{P}_{2} \mathrm{O}_{5} \mathrm{ha}^{-1}$, contudo, as maiores taxas de eficiência de utilização do fósforo foram constatadas com níveis de fertilização entre 50 e $100 \mathrm{~kg}$ de $\mathrm{P}_{2} \mathrm{O}_{5} \mathrm{ha}^{-1}$. No entanto, Faria et al. (2015), em pastagens de $M$. maximus cv. Mombaça recebendo adubação de manutenção (45 $\mathrm{kg}$ de $\mathrm{N} \mathrm{ha}^{-1}$ e $40 \mathrm{~kg}$ de $\mathrm{K}_{2} \mathrm{O}$ ha $^{-1}$ ) observaram efeito linear da adubação fosfatada $(0,35,70,140$ e $280 \mathrm{~kg}$ de $\mathrm{P}_{2} \mathrm{O}_{5} \mathrm{ha}^{-1}$ ), enquanto que sem adubação de manutenção o máximo rendimento de MSV foi obtido com a aplicação de $218 \mathrm{~kg}$ de $\mathrm{P}_{2} \mathrm{O}_{5}$ $\mathrm{ha}^{-1}$. Para a mesma gramínea, Oliveira et al. (2012) e Carneiro et al. (2017) estimaram em 215,1 e 
$273,2 \mathrm{~kg}$ de $\mathrm{P}_{2} \mathrm{O}_{5} \mathrm{ha}^{-1}$ as doses de máxima eficiência técnica para a produtividade de MSV.

Os teores de $\mathrm{P}$ foram ajustados ao modelo quadrático de regressão e o máximo valor obtido com a aplicação de 74,91 kg de $\mathrm{P}_{2} \mathrm{O}_{5}$ ha $^{-1}(1,87 \mathrm{~g}$ $\mathrm{kg}^{-1}$ ), contudo foram superiores aos reportados por Costa (2004) para pastagens de M. maximus cv. Tanzânia fertilizadas com $40 \mathrm{~kg}$ de $\mathrm{N} \mathrm{ha}^{-1}, 60 \mathrm{~kg}$ de $\mathrm{P}_{2} \mathrm{O}_{5}$ ha $^{-1}$ e $40 \mathrm{~kg}$ de $\mathrm{K}_{2} \mathrm{O} \mathrm{ha}^{-1}$. O NCI de $\mathrm{P}$, relacionado com $80 \%$ da produção máxima de
MSV, foi estimado em 1,692 $\mathrm{g} \mathrm{kg}^{-1}$ e obtido com a aplicação de $34,8 \mathrm{~kg}$ de $\mathrm{P}_{2} \mathrm{O}_{5}$ ha $^{-1}$, sendo inferior aos reportados por Costa et al. (2007) para $M$. maximus cvs. Tobiatã $\left(1,97 \mathrm{~g} \mathrm{~kg}^{-1}\right)$, Centenário $\left(1,93 \mathrm{~g} \mathrm{~kg}^{-1}\right)$ e Vencedor $\left(1,84 \mathrm{~g} \mathrm{~kg}^{-1}\right)$, evidenciando elevada eficiência de utilização de $\mathrm{P}$ na produção de forragem, pois o NCI representa a concentração do nutriente abaixo da qual o rendimento é reduzido e acima não apresenta retorno econômico (Nabinger and Carvalho, $\underline{2009)}$.

Tabela 1. Rendimento de matéria seca verde (MSV - $\mathrm{kg} \mathrm{ha}^{-1}$ ), eficiência de utilização do fósforo (EUP - $\mathrm{kg}$ de MSV/kg de $\mathrm{P}_{2} \mathrm{O}_{5}$ ha $\left.^{-1}\right)$, teor de fósforo $\left(\mathrm{g} \mathrm{kg}^{-1}\right)$, densidade populacional de perfilhos $\mathrm{m}^{-2}$ (DPP), número de folhas perfilho-1 (NFP), tamanho médio de folhas (TMF - cm), índice de área foliar (IAF), taxa de aparecimento de folhas (TAF - folhas perfilho ${ }^{-1} \mathrm{dia}^{-1}$ ), taxa de expansão foliar (TEF - cm perfilho ${ }^{-1} \mathrm{dia}^{-1}$ ) e taxa de senescência foliar (TSF - cm perfilho-1 $\mathrm{dia}^{-1}$ ) de Megathyrsus maximus cv. Mombaça, em função da adubação fosfatada.

\begin{tabular}{ccccccc}
\hline \multirow{2}{*}{ Variáveis } & \multicolumn{5}{c}{ Doses de $\mathrm{P}_{2} \mathrm{O}_{5} \mathrm{ha}^{-1}$} & \multirow{2}{*}{ Equação de Regressão } \\
\cline { 2 - 6 } & 0 & 30 & 60 & 90 & 120 & \\
\hline MSV & 2.389 & 2.987 & 3.451 & 3.698 & 3.533 & $\mathrm{Y}=2.362+26,597 \mathrm{X}-0,1383 \mathrm{X}^{2}\left(\mathrm{R}^{2}=0,92\right)$ \\
EUP & --- & 99,57 & 57,52 & 41,09 & 29,44 & $\mathrm{Y}=113,6-0,756 \mathrm{X}\left(\mathrm{r}^{2}=0,90\right)$ \\
Teor de P & 1,47 & 1,73 & 1,89 & 1,81 & 1,74 & $\mathrm{Y}=1,475+0,010638 \mathrm{X}-0,000071 \mathrm{X}^{2}\left(\mathrm{R}^{2}=0,91\right)$ \\
DPP & 189 & 228 & 357 & 371 & 342 & $\mathrm{Y}=171,7+3,8871 \mathrm{X}-0,01999 \mathrm{X}^{2}\left(\mathrm{R}^{2}=0,88\right)$ \\
NFP & 3,51 & 4,03 & 4,81 & 5,07 & 4,72 & $\mathrm{Y}=3,824+0,0284 \mathrm{X}-0,000221 \mathrm{X}^{2}\left(\mathrm{R}^{2}=0,83\right)$ \\
IAF & 2,11 & 2,76 & 2,98 & 3,37 & 2,71 & $\mathrm{Y}=2,07+0,02936 \mathrm{X}-0,000194 \mathrm{X}^{2}\left(\mathrm{R}^{2}=0,91\right)$ \\
TMF & 27,16 & 30,11 & 33,54 & 34,98 & 31,22 & $\mathrm{Y}=26,61+0,1901 \mathrm{X}-0,00123 \mathrm{X}^{2}\left(\mathrm{R}^{2}=0,91\right)$ \\
TAF & 0,084 & 0,101 & 0,109 & 0,097 & 0,092 & $\mathrm{Y}=0,084+0,000633 \mathrm{X}-0,0000052 \mathrm{X}^{2}\left(\mathrm{R}^{2}=0,89\right)$ \\
TEF & 2,28 & 3,02 & 3,66 & 3,37 & 2,89 & $\mathrm{Y}=2,24+0,03732 \mathrm{X}-0,000267 \mathrm{X}^{2}\left(\mathrm{R}^{2}=0,94\right)$ \\
TSF & 0,107 & 0,139 & 0,158 & 0,162 & 0,174 & $\mathrm{Y}=0,1168+0,000532 \mathrm{X}\left(\mathrm{r}^{2}=0,88\right)$ \\
\hline
\end{tabular}

Para a DPP, NFP, IAF e TMF as relações foram ajustadas ao modelo quadrático de regressão e os máximos valores obtidos com a aplicação de 97,5; 64,4; 75,6 e 79,2 kg de $\mathrm{P}_{2} \mathrm{O}_{5}$ ha 1 , respectivamente. As correlações entre rendimento de MS e NPP $(r=0,9132 ; \mathrm{P}=0,0021)$ e NFP $(r=0,9411 ; \mathrm{P}=0,0017)$ foram positivas e significativas, as quais explicaram em 83,4 e $88,56 \%$, respectivamente, os incrementos verificados nos rendimentos de forragem da gramínea, em função da adubação fosfatada. Os valores registrados, neste trabalho, para o NP, NFP, TMF e IAF foram superiores aos reportados por Costa (2004) para pastagens de M. maximus cv. Tobiatã, fertilizadas com $60 \mathrm{~kg}$ de $\mathrm{P}_{2} \mathrm{O}_{5} \mathrm{ha}^{-1} \mathrm{e}$ submetidas a cortes a cada 35 dias, que estimaram 207 perfilhos $\mathrm{m}^{-2} ; 3,82$ folhas perfilho ${ }^{-1} ; 23,6 \mathrm{~cm}$ folha $^{-1}$ e IAF de 2,36.
Em pastagens de $M$. maximus cv. Tanzânia, Patês et al. (2007) constataram efeito positivo da adubação fosfatada sobre a DPP, sendo o máximo valor obtido com a aplicação de $120 \mathrm{~kg}$ de $\mathrm{P}_{2} \mathrm{O}_{5}$ ha $^{-1}$; contudo para o NFP a relação foi linear e negativa. $\mathrm{O}$ perfilhamento da gramínea depende da velocidade de emissão de folhas que produzem gemas aptas a originar novos perfilhos, dependendo das condições ambientais e práticas de manejo adotadas (Lemaire et al., 2011). Em relvados densos e com elevada disponibilidade de forragem, o ambiente luminoso afeta a competição entre plantas, por meio da quantidade e qualidade de luz incidente e da razão vermelho:vermelho distante. Quando a luz penetra no dossel, atenuase a luz vermelha e a que alcança os estratos inferiores da planta é predominantemente vermelho extremo, caracterizada como fotossinteticamente ineficiente, promovendo redução na produção de perfilhos, notadamente os 
de ordem mais elevada (Ballaré et al., 1987). Em geral, a DPP incrementa até o ponto em que não há competição entre perfilhos, pois a partir do momento em que há competição intraespecífica, sua população se estabiliza e para cada perfilho que surge, ocorre senescência de outro. O IAF representa a síntese das características morfogênicas e estruturais da gramínea e reflete o balanço dos processos que determinam a oferta (fotossíntese) e a demanda (respiração, acúmulo de reservas, síntese e senescência de tecidos) de fotoassimilados, que estabelecem o ritmo de crescimento da pastagem (Nabinger and Carvalho, 2009). Os efeitos da adubação fósforo sobre a TAF e TEF foram ajustados ao modelo quadrático de regressão e os máximos valores obtidos com a aplicação de 58,6 e 69,8 kg de $\mathrm{P}_{2} \mathrm{O}_{5}$ ha $^{-1}$ (Tabela 1). A TAF e a TEF apresentam correlação negativa, indicando que quanto maior a TAF, menor será o tempo disponível para o alongamento das folhas (Cecato et al., 2007, Costa et al., 2012). Neste trabalho, a correlação entre as duas variáveis foi positiva e significativa $(\mathrm{r}=$ 0,8629; $\mathrm{P}=0,0049$ ), possivelmente como consequência da maior fertilidade do solo, a qual contribuiu positivamente para a maximização das características morfogênicas da gramínea, além das avaliações que foram realizadas a intervalos constantes. Quadros et al. (2005) reportaram maiores TAF e TEF para Paspalum urvillei com a aplicação conjunta de $100 \mathrm{~kg}$ de $\mathrm{P}_{2} \mathrm{O}_{5}$ ha $^{-1}$ e 100 $\mathrm{kg}$ de $\mathrm{K}_{2} \mathrm{O}$ ha $^{-1}$ (0,106 folhas perfilho ${ }^{-1} \mathrm{dia}^{-1} \mathrm{e}$ $\left.0,376 \mathrm{~mm}_{\text {folha }}{ }^{-1} \mathrm{dia}^{-1}\right)$, comparativamente a aplicação de $50 \mathrm{~kg}$ de $\mathrm{P}_{2} \mathrm{O}_{5}$ ha-1 e $50 \mathrm{~kg}$ de $\mathrm{K}_{2} \mathrm{O}$ ha${ }^{1}\left(0,082\right.$ folhas perfilho ${ }^{-1}$ dia $^{-1}$ e $0,279 \mathrm{~mm}_{\text {folha }}{ }^{-1}$ $\operatorname{dia}^{-1}$ ). Lemaire et al. (2011) observaram correlação positiva entre TEF e quantidade de folhas verdes remanescentes no perfilho após a desfolhação, sendo o tamanho do perfilho o responsável pela longa duração da TEF. Neste trabalho, a correlação foi positiva e significativa ( $\mathrm{r}$ $=0,9421 ; \mathrm{P}=0,0019)$, evidenciando a sincronia entre estas duas variáveis. A TEF, em decorrência de sua alta correlação com a produção de MS, tem sido utilizada como critério para a seleção de gramíneas em trabalhos de melhoramento genético (Nabinger and Carvalho, 2009); enquanto que a TAF é a característica morfogênica de maior destaque, uma vez que afeta diretamente as três características estruturais do relvado: tamanho da folha, densidade de perfilhos e número de folhas perfilho ${ }^{-1}$ (Cecato et al., 2007, Santos et al., 2012).
As TSF foram diretamente proporcionais às doses de fósforo, refletindo a aceleração do processo de renovação de tecidos como consequência da maior produtividade de forragem (Tabela 1). Os valores observados foram inferiores aos reportados por Costa (2004) para $M$. maximus cv. Tanzânia que estimou TSF de 0,201 e $0,267 \mathrm{~cm}_{\text {perfilho }}{ }^{-1} \mathrm{dia}^{-1}$, para plantas avaliadas aos 49 e 56 dias de rebrota, respectivamente. Costa et al. (2007) avaliando genótipos de Megathyrsus, reportaram maiores TSF com a aplicação de 60 $\left(0,188 \mathrm{~cm}_{\text {perfilho }}{ }^{-1} \mathrm{dia}^{-1}\right)$ ou $120 \mathrm{~kg}$ de $\mathrm{P}_{2} \mathrm{O}_{5} \mathrm{ha}^{-1}$ $\left(0,198 \mathrm{~cm}\right.$ perfilho $\left.\mathrm{dia}^{-1}\right)$, comparativamente a 30 $\mathrm{kg}$ de $\mathrm{P}_{2} \mathrm{O}_{5} \mathrm{ha}^{-1}\left(0,107 \mathrm{~cm}\right.$ perfilho $\left.{ }^{-1} \mathrm{dia}^{-1}\right)$. A senescência é o processo natural que caracteriza a última fase de desenvolvimento de uma folha. Iniciado após a completa expansão das primeiras folhas é intensificado com o aumento da área foliar o que implica no sombreamento das folhas inseridas na porção inferior do colmo (Lemaire et al., 2011). A senescência apesar do efeito negativo sobre a qualidade da forragem representa importante processo fisiológico no fluxo de tecidos da gramínea, pois em torno de 35; 68; 86 e $42 \%$ do nitrogênio, fósforo, potássio e magnésio, respectivamente, podem ser reciclados das folhas senescentes e utilizados para a produção de novos tecidos foliares (Sarmiento et al., 2006, $\underline{\text { Costa et }}$ al., 2013).

\section{Conclusões}

A adubação fosfatada afeta positivamente a disponibilidade de forragem e as características morfogênicas e estruturais da gramínea. A eficiência de utilização do fósforo é inversamente proporcional às doses aplicadas. $\mathrm{O}$ processo de renovação e senescência de tecidos da gramínea são acelerados com o aumento das doses de fósforo.

\section{Referências Bibliográficas}

Ballaré, C. L., Sánchez, R. A., Scopel, A. L. \& Ghersa, C. 1987. Early detection of neighbour plants by phytochrome perception of spectral changes in reflected sunlight. Plant, Cell \& Environment, 10, 551-557.

Barger, N. N., D'Antonio, C. M., Ghneim, T., Brink, K. \& Cuevas, E. 2002. Nutrient limitation to primary productivity in a secondary savanna in Venezuela. Biotropica, 34, 493-501.

Carneiro, J. S. S., Silva, P. S. S., Santos, A. C. M., Freitas, G. A. \& Silva, R. R. 2017. Response of grass Mombasa under the effect of sources and 
doses of phosphorus in the fertilization formation. Journal of Bioenergy and Food Science, 4, 12-25.

Cecato, U., Skrobot, V. D., Fakir, G. M., Jobim, C. C., Branco, A. F., Galbeiro, S. \& Janeiro, V. 2007. Características morfogênicas do capim mombaça (Panicum maximum cv. Mombaça) adubado com fontes de fósforo, sob pastejo. Revista Brasileira de Zootecnia, 36, 1699. 1706.

Costa, N. L. 2004. Formação, manejo e recuperação de pastagens em Rondônia. Porto Velho: Embrapa Rondonia. 217p.

Costa, N. L., Gianluppi, V., Braga, R. M. \& Bendahan, A. B. 2009. Alternativas tecnológicas para a pecuária de Roraima. Boa Vista: Embrapa Roraima. 35p. (Documentos, 19).

Costa, N. L., Magalhães, J. A., Pereira, R. G. A., Townsend, C. R. \& Oliveira, J. R. C. 2007. Considerações sobre o manejo de pastagens na Amazônia Ocidental. Revista do Conselho Federal de Medicina Veterinária, 40, 37-56.

Costa, N. L., Moraes, A., Gianluppi, V. \& Magalhães, J. A. 2012. Rendimento de forragem e morfogênese de Axonopus aureus, durante o período seco, nos cerrados de Roraima.Revista Agro@mbiente, 6, 59-66.

Costa, N. L., Moraes, A., Monteiro, A. L. G., Motta, A. C. V., Oliveira, R. A. \& Rodrigues, A. N. A. 2013. Forage productivity and morphogenesis of Axonopus aureus under different nitrogen fertilization rates. Revista Brasileira de Zootecnia, 42, 541-548.

Costa, N. L., Townsend, C. R., Magalhães, J. A., Paulino, V. T., Rodrigues, A. N. A. \& Rodrigues, B. H. N. 2015. Resposta de Panicum maximum cv. Massai à fertilização fosfatada. Pubvet: Publicações em Medicina Veterinaria e Zootecnia, 9, 429-466.

Faria, Á. J. G., Freitas, G. A., Georgetti, A. C. P., Júnior, J. M. F., Silva, M. C. A. \& Silva, R. R. 2015. Adubação nitrogenada e potássica na produtividade do capim Mombaça sobre adubação fosfatada. Journal of Bioenergy and Food Science, 2, 98-106.

Lemaire, G. \& Agnusdei, M. 2000. Leaf tissue turnover and efficiency of herbage utilization. In: Lemaire, G., Hodgson, J., Moraes, A.,
Carvalho, P. C. F. \& Nabinger, C. (Eds.) Grassland ecophysiology and grazing ecology. $\mathrm{CAB}$ International Publishing, New York. p.265-287.

Lemaire, G. \& Chapman, D. 1996. Tissue flows in grazed plant communities. In: Hodgson, J. \& Illius, A. W. (Eds.) The ecology and management of grazing systems. $\mathrm{CAB}$ International, Wallingford. p.3-36.

Lemaire, G., Hodgson, J. \& Chabbi, A. 2011. Grassland productivity and ecosystem services. Cabi, Wallingford. 297p.

Nabinger, C. \& Carvalho, P. C. F. 2009. Ecofisiología de sistemas pastoriles: aplicaciones para su sustentabilidad. Agrociencia, 13, 18-27.

Oliveira, P. S. R., Deminicis, B. B., Castagnara, D. D. \& Gomes, F. C. N. 2012. Efeito da adubação com fósforo do capim Mombaça em solos com texturas arenosa e argilosa. Archivos de Zootecnia, 61, 397-406.

Patês, N. M. S., Pires, A. J. V., Silva, L. C. S., Carvalho, G. G. P. \& Freire, M. L. A. 2007. Características morfogênicas e estruturais do capim-tanzânia submetido a doses de fósforo e nitrogênio. R. Bras. Zootec, 36, 1736-1741.

Quadros, F. L. F., Bandinelli, D. G., Pigatto, A. G. S. \& Rocha, M. G. 2005. Morfogênese de Lolium multiflorum Lam. e Paspalum urvillei Steud sob níveis de adubação de fósforo e potássio. Ciência Rural, 35, 181-186.

Santos, M. R., Fonseca, D. M., Gomes, V. M., Silva, S. P., Silva, G. P. \& Reis, M. 2012. Correlações entre características morfogênicas e estruturais em pastos de capim-braquiária. Ciência Animal Brasileira, 13, 49-56.

Sarmiento, G., Silva, M. P., Naranjo, M. E. \& Pinillos, M. 2006. Nitrogen and phosphorus as limiting factors for growth and primary production in a flooded savanna in the Venezuelan Llanos. Journal of Tropical Ecology, 22, 203-212.

Article History:

Received 30 June 2017

Accepted 20 July 2017

Available on line 8 September 2017

License information: This is an open-access article distributed under the terms of the Creative Commons Attribution License 4.0, which permits unrestricted use, distribution, and reproduction in any medium, provided the original work is properly cited 